\title{
Artemisin Combined Therapy in malaria patients: Do we need to search for more?
}

\author{
Jani C. ${ }^{1}$, Palkhiwala N. ${ }^{2}$, Parikh A. ${ }^{3}$, Suthar N. ${ }^{4}$, Patel K. ${ }^{5}$ \\ ${ }^{1}$ Dr. Chinmay Jani, Intern, ${ }^{2}$ Dr. Neil Palkhiwala, Resident Doctor, Internal Medicine, ${ }^{3}$ Dr. Ami Parikh, Professor \& HOD, \\ Internal Medicine, above authors are affiliated with Smt NHL Municipal Medical College, Ahmedabad, Gujarat, India, \\ ${ }^{4}$ Dr. Nilay Suthar, Professor, Internal Medicine, AMCMET Medical College, Ahmedabad, Gujarat, India, ${ }^{5}$ Dr. Khushali \\ Patel, Assistant Professor, Internal Medicine, Smt NHL Municipal Medical College, Ahmedabad, Gujarat, India.
}

Corresponding Author: Dr. Chinmay Jani, Intern, Smt NHL Municipal Medical College, Ahmedabad, Gujarat, India.

\begin{abstract}
Introduction: Malaria is a vector borne disease highly prevalent in the topical developing countries. Two main species of plasmodium causing majority of diseases manifestations are P. Vivax and P. falciparum. The approach to antimalarial selection is determined based on the location of the patient. For treatment of uncomplicated P. falciparum malaria, according to WHO guidelines first line therapy mainly includes Artemisin Combined Therapy. Concerns about the emergence of resistance to artemisin derivatives have increased recently. The main aim of the present study is to investigate the effect of using a combination of intravenous artesunate along with oral doxycycline, as a novel ACT for Malarial infections - falciparum vs. vivax. Methodology: Prospective observational study was carried out at V S General Hospital in Ahmedabad, Gujarat, India. A full history of current illness was taken followed by examining the serial peripheral smear reports of the patients till the malarial parasites are not seen on two consecutive occasions. Samples were taken at least 6 hours apart by the capillary method and oral temperature was measured every 6 hours. We excluded other associated viral fevers such as dengue, pediatric age group ( $<12$ years) and Co-Morbid illnesses like hepatic or renal dysfunction. Data analysis was done using SPSS software version 20. Results: Student t test showed that PCT was significantly more in falciparum (mean= 74.53 hours) patients as compared to vivax patients (mean= 51.89 hours). $(\mathrm{p}<0.001)$. Also duration of stay was significantly more in patients having falciparum (mean $=3.63$ days) as compared to patients having vivax (mean $=2.32$ days $)(\mathrm{p}<0.001)$ Multivariate analysis by linear regression showed that species of the parasite was the most significant independent predictor $(B=12.552)$ of the time to parasite clearance and other significant variable was Grade of parasitemia at 0 hour $(B=12.798)$. We also found that patients with residual parasitemia was $83.78 \%, 40.54 \%$ and $10.81 \%$ in vivax group whereas it was $100 \%, 88.15 \%$ and $46.05 \%$ in falciparum respectively at 24 , 48 and 72 hours. Conclusion: The study shows that PCT and residual parasitemia is very high in falciparum patients as compared to previous reports of different studies and also as compared to vivax group patients. ACT resistance is a grave concern for falciparum and more studies should be done to understand pathophysiology and its prevalence in India. We strongly suggest that a continues monitoring needs to be implemented in health policy to understand the dynamicity of emerging resistance
\end{abstract}

Keywords: Malaria, Artemisin Combined Therapy, Resistance, Artesunate, Doxycycline

\section{Introduction}

Malaria is a vector borne disease highly prevalent in the topical developing countries. It accounts for an estimated 250 to 500 million febrile illnesses and up to a million deaths annually. The World Malaria Report 2017 by the World Health Organization says that global malaria cases have declined steadily since 2010 (237

Manuscript received: $25^{\text {th }}$ August 2018

Reviewed: $4^{\text {th }}$ September 2018

Author Corrected: $9^{\text {th }}$ September 2018

Accepted for Publication: $13^{\text {th }}$ September 2018 million), but there was a significant increase in the number of cases in 2016 (216 million) compared with 2015 (211 million) [2] [1]. Two main species of plasmodium causing majority of diseases manifestations are P. Vivax and P. falciparum. Clinical manifestations vary based on species, age of the patient and immunity of the patient along with major contribution of the environmental factors. Malaria should always be considered as one of the main cause of any febrile illness in the endemic regions. Initial symptoms are 


\section{Original Research Article}

mainly non-specific including tachycardia, fever associated with chills, malaise, nausea, abdominal pain, diarrhea, myalgias and arthralgias. P.falciparum malaria can be much more acute and severe than malaria caused by other Plasmodium species. Although P.Vivax can cause serious and fatal illness, by far the largest fraction of deaths directly attributable to malaria are caused by severe complications of P. falciparum infection, including cerebral malaria, severe anemia, respiratory failure, renal failure, and severe malaria of pregnancy [3].

The approach to antimalarial selection is determined by whether infection was acquired $\mathrm{u}$ area with Chloroquine sensitivity or resistance. In general treatment of uncomplicated malaria consists of oral therapy with combination of two agents (in case of CQ resistance) or $\mathrm{Cq}$ monotherapy in case of CQ sensitive. For treatment of uncomplicated P. falciparum malaria, according to WHO guidelines first line therapy mainly includes Artemisin Combined Therapy [4]. ACTs have a low side effect profile, are potent against all stages of plasmodium and have the most rapid clearance time relative to other antimalarial drugs [5]. Artemisins should always be administered with second line agent that has a longer half life than the Artemisin drug to provide an extended duration of drug level to clear parasitemia $[6,7]$.

Artemisin resistance has been observed starting mainly from South-east Asia with use of monotherapy being the main cause [8]. Concerns about the emergence of resistance to artemisin derivatives have increased recently with reports of treatment failures with artesunate-mefloquine and artemether-lumefantrine in Thai and Cambodian malaria control programs [9]. These failures may be associated with relatively slower elimination of parasites in response to artemisin derivatives in vivo (prolonged clearance times), which invokes the specter of current artemisin combination therapy (ACT) regimens becoming less effective. They also raise the possibility that rapid parasite clearance, a hallmark benefit of artemisinins in the treatment of severe malaria, may become less dependable after artemisinin dosing in Southeast Asia [10].

Aim: The main aim of the present study is to investigate the effect of using a combination of intravenous artesunate along with oral doxycycline, as a novel ACT for Malarial infections - falciparum vs. vivax and to compare the clinical and parasitological results of our study with different studies in Asia and other parts of the world.

\section{Other objectives of the study include}

1. To compare the Parasite Clearance time (PCT) in both the groups.

2. To compare the duration of stay and fever clearance time in both the groups.

3. To find out the variable factor affecting PCT the most amongst all the variables.

\section{Methodology}

Study type and design: Prospective observational study

Study site: V.S General Hospital, Ahmedabad, Gujarat, India.

After taking approval of the Institutional Review Board, we started the study with all adult patients (aged 12-80 years), having malarial infection, proven by peripheral smear or rapid diagnostic kit testing who were admitted to our hospital. Written informed consent was taken from all the patients.

A full history of current illness as well as past history was obtained followed by examining the serial peripheral smear reports of the patients till the malarial parasites are not seen on two consecutive occasions. Samples were taken at least 6 hours apart by the capillary method and oral temperature was measured every 6 hours. Fever clearance times were defined as the time of the first temperature reading of less than 37.5 ' $\mathrm{c}$ and the time to the start of the first 24- hour period during which the temperature remained below 37.5 'c. Parasitic clearance time was defined as the time from the start of treatment until malarial parasites are not seen in two consecutive occasions. Patients were assessed weekly till 1 month after discharge and were advised to return if they felt unwell. Peripheral Smear analysis was done on each follow up visit of these patients.

\section{Inclusion criteria}

- Malaria parasite positive for vivax or falciparum

\section{Exclusion criteria}

- Other associated viral fevers such as dengue

- Pediatric age group $(<12$ years $)$

- Co-Morbid illnesses like hepatic or renal dysfunction.

Data analysis was done using SPSS software version 20. We used t-test for finding out the significance of difference between results of vivax and falciparum group. Regression analysis was done for carrying out multi-variate analysis. 


\section{Results}

\section{Descriptive statistics}

Total data of 224 patients was collected between August 2015 to April 2016

Out of 224, 148 patients had vivax and 76 had falciparum.

Out of 224, 112 were males and 112 were females. Mean age of patients was 36.54 years with Standard Deviation of 14.48 years.

On follow up visits, peripheral smear analysis was done for all the patients and all these patients had negative results at all their follow up visits.

Table-1: Clinical and parasitological response in two study groups.

\begin{tabular}{|c|c|c|c|}
\hline Groups: & Total & Vivax & Falciparum \\
\hline \multicolumn{4}{|l|}{ Duration of stay } \\
\hline Mean & 2.77 & 2.32 & 3.63 \\
\hline Median & 2.5 & 2 & 4 \\
\hline Standard Deviation & 1.227 & 0.843 & 1.394 \\
\hline \multicolumn{4}{|c|}{ Days of fever before presentation } \\
\hline Mean & 2.19 & 2.16 & 2.24 \\
\hline Median & 2 & 2 & 2 \\
\hline Standard Deviation & 1.253 & 1.212 & 1.335 \\
\hline \multicolumn{4}{|c|}{ Duration of fever after therapy } \\
\hline Mean & 1.56 & 1.31 & 2.05 \\
\hline Median & 1 & 1 & 2 \\
\hline Standard Deviation & 0.886 & 0.545 & 1.176 \\
\hline \multicolumn{4}{|c|}{ Grade of parasitemia at 0 hours } \\
\hline Mean & 2.68 & 2.46 & 3.11 \\
\hline Median & 3 & 2 & 3 \\
\hline Standard Deviation & 0.91 & 0.95 & 0.645 \\
\hline \multicolumn{4}{|c|}{ Parasitic Index at 0 hour } \\
\hline Mean & 1.575 & 0.892 & 2.905 \\
\hline Median & 0.7 & 0.5 & 1 \\
\hline Standard Deviation & 2.7136 & 0.9841 & 4.1573 \\
\hline \multicolumn{4}{|c|}{ Parasitic Clearance time } \\
\hline Mean & 59.57 & 51.89 & 74.53 \\
\hline Median & 60 & 48 & 72 \\
\hline Standard Deviation & 22.149 & 18.154 & 21.672 \\
\hline
\end{tabular}

The mean number of days of fever before enrollment was 2.19 with SD: 1.25 . It was similar in both the groups of patient with Mean in vivax $=2.16$ days and in falciparum $=2.24$. 
Original Research Article

Table-2: Residual parasitemia.

\begin{tabular}{|c|c|c|c|}
\hline Residual Parasitemia & Total & Vivax & Falciparum \\
\hline 24 hours & 200 & 124 & 76 \\
\hline 48 hours & 127 & 60 & 35 \\
\hline 72 hours & 51 & 16 & 76 \\
\hline Total patients & 224 & 148 & 67 \\
\hline
\end{tabular}

We also found that patients with residual parasitemia at day 1 was $83.78 \%$ in vivax group whereas all $100 \%$ patients in falciparum had residual parasitemia at 24 hours.

At 48 hours it was reduced to $40.54 \%$ in vivax group whereas falciparum group still had $88.15 \%$ who had parasitemia.

At 72 hours majority of vivax group patients had cleared parasitemia with residual parasitemia only being positive in $10.81 \%$ where as falciparum group still had residual parasitemia in $46.05 \%$ patients.

We compared the results of other variables between two groups using student $t$ test.

Table-3: Comparison of PCT, duration of stay at the hospital, duration of fever after therapy between two groups by t-test.

\begin{tabular}{|c|c|c|c|c|c|}
\hline & T & Df & Sig. (2-tailed) $\mathbf{p}$ & \multicolumn{2}{|c|}{$\mathbf{9 5 \% \text { Confidence Interval of the Difference }}$} \\
\cline { 5 - 6 } & & & value & Lower & Upper \\
\hline Pct & -8.262 & 222 & $<.001$ & -28.034 & -17.235 \\
\hline Duration of stay & -8.727 & 222 & $<.001$ & -1.602 & -1.012 \\
\hline $\begin{array}{c}\text { Duration of fever } \\
\text { after therapy }\end{array}$ & -6.449 & 222 & $<.001$ & -.969 & -.515 \\
\hline
\end{tabular}

Based on the above mentioned student t test, PCT was significantly more in falciparum (mean= 74.53 hours) patients as compared to vivax patients (mean= 51.89 hours). Also duration of stay was significantly more in patients having falciparum (mean $=3.63$ days) as compared to patients having vivax (mean $=2.32$ days) and duration of fever after therapy was also more in falciparum patients (mean=2.05 days) as compared to patients having vivax (mean $=1.31$ days).

Further analysis was done using linear regression with parasitic clearance time as dependant variable and independent variables included species of malaria, parasitic index at 0 hour, sex, age, days of fever before presentation, grade of parasitemia at 0 hour.

Table-4: Linear Regression analysis.

\begin{tabular}{|c|c|c|c|c|c|}
\hline \multirow[t]{2}{*}{ Model } & \multicolumn{2}{|c|}{ Unstandardized Coefficients } & \multirow{2}{*}{$\begin{array}{c}\begin{array}{c}\text { Standardized } \\
\text { Coefficients }\end{array} \\
\text { Beta }\end{array}$} & \multirow[t]{2}{*}{$\mathbf{T}$} & \multirow[t]{2}{*}{ Sig. } \\
\hline & $\mathbf{B}$ & Std. Error & & & \\
\hline (Constant) & 3.602 & 5.713 & & .631 & .529 \\
\hline Age & .286 & .070 & .187 & 4.089 & .000 \\
\hline Sex & -2.751 & 1.937 & -.062 & -1.420 & .157 \\
\hline Species & 12.552 & 2.239 & .269 & 5.606 & .000 \\
\hline $\begin{array}{c}\text { Days of fever before } \\
\text { presentation }\end{array}$ & -.940 & .801 & -.053 & -1.175 & .241 \\
\hline $\begin{array}{c}\text { Grade of parasitemia at } 0 \\
\text { hour }\end{array}$ & 12.798 & 1.277 & .526 & 10.020 & .000 \\
\hline Parasitic Index at 0 hour & .377 & .435 & .046 & .866 & .387 \\
\hline
\end{tabular}

Dependent Variable: pct 


\section{Original Research Article}

Above results showed that species was a significant independent predictor ( $\mathrm{B}=12.552)$ of the time to parasite clearance.). Other significant variable was Grade of parasitemia at 0 hour $(B=12.798)$. Also as seen in the above table $\mathrm{R}$ square is 0.587 which shows that $58.7 \%$ of total variance in the PCT is explained by the above included variables. This shows that Falciparum is the major factor for increased pct.

\section{Discussion}

In our cross sectional study we tried to study various parasitological and clinical presentations in the patients having vivax or falciparum taking Artemisin combined therapy including: IV artesunate with oral doxycycline. Artesunate is a semi synthetic derivative of Artemisin. It is a prodrug which is converted to dihydroartemisin (DHA). DHA is an antimalarial agent active against all of the eryhtrocytic stage of the parasite including gametocytes; inhibits parasite metabolism and enhances the clearance of the infected erythrocytes.

Artesunate is highly efficacious in treatment of severe malaria and is most of the time combined with oral drugs like Doxycycline or Atovaquone- proguanil. In general, Artemisins should never be used as a monotherapy due to increased drug resistance [9].

ACT combines the highly effective short acting Artemisins with a longer acting partner to protect against Artemisin resistance. Some of the studies have even observed that oral therapy if not observed and followed properly can lead to treatment failure [11]. In our study we observed that ACT was more effective in clearing the parasite from the blood in case of vivax as compared to falciparum. Although many factors depend on the PCT, pathophysiology of the disease also being one of them. Nevertheless the effect of drug should not be ignored at all.

Artemisin resistance is becoming a serious problem in most of the malaria endemic countries in the world. Artemisin resistance has started appearing in South east Asia region since 2003. Many studies have started focusing on the genotypic mechanism of the resistance. In a study by Thita et al it was found that approximately $94 \%$ of the isolates collected from 2009 to 2016 contained the pfmdr1 $184 \mathrm{~F}$ allele.

Mutations of the k13 gene were detected in approximately $90 \%$ of the parasites collected from 2009 to 2016 which were significantly higher than the parasite isolates collected before [12].

In an another study by Singh et al they found that Mutations in Plasmodium falciparum gene kelch13 (pfkelch13) are strongly and causally associated with resistance to anti-malarial drug artemisin [13].
India is having a very high incidence of malaria patients every year. Every year the prevalence of co-infection with Dengue and Chickungunya is also increasing in India which leads to increased morbidity and mortality in such patients [14]. Very few studies have focused on the resistance of the antimalarial agents in India. Sehgal et al first reported Chloroquine resistance in $\mathrm{P}$. falciparum in Assam state of India [15].

This was followed by many reports of Chloroquine resistance in P. falciparum from various other parts of the country like Odisha, Madhya Pradesh, Gujarat and the north-eastern region of the nation [16]. Very few cases of Artemisin resistance have been reported in India although many different studies on resistance of other antimalarial therapies have raised these concerns. Also resistance in the neighboring nations have also alarmed the medical faculty of India [17,18]. Although through our study we cannot prove that there is resistance to Artemisin in the falciparum patients but we can surely observe decreased efficacy of the treatment in the falciparum patients. More detailed in-vitro and invivo analysis of resistance can give a complete picture of the current scenario in the nation.

In our study mean of Parasite Clearance Time was found to be 59.57 hours in overall study, 51.89 hours in patients having vivax and 74.53 hours in patients having falciparum. Different studies have observed different PCT. In a study by Dondorp et al they had compared parasite Parasite clearance between falciparum patients of two different towns of Cambodia and Thailand using a randomized control trial.

They had divided patients into two groups, one group with monotherapy of Artesunate and other group having combined therapy of Artesunate with other oral drugs.

They found that it was markedly slower in Pailin, Cambodia with a median parasite clearance time for both Pailin treatment groups combined of 84 hours as compared with 48 hours in Wang Pha, Thailand groups $(\mathrm{P}<0.001)$. Our study shows that patients with falciparum were having plasma clearance time of 74.53 hours which was almost similar to the Cambodian town which raised our concern that there might be development of resistance in Indian population too [9]. 


\section{Original Research Article}

Another study by Bharti et al focused on efficacy of Artemether- Lumefantrine in the treatment of falciparum malaria. The study showed that the mean fever clearance time was $27.2 \mathrm{~h}+/-8.2(24-48 \mathrm{~h})$ and the mean parasite clearance time was $30.1 \mathrm{~h}+/-11.0$ (24-72 h) and they hence proved that the drug was efficacious. While comparing our results to this study, we can see that mean parasite clearance time was 74.53 hours +/- 21.62 which is very high as compared to their PCT [19].

We also found that residual parasitemia was We also found that patients with residual parasitemia was $83.78 \%, 40.54 \%$ and $10.81 \%$ in vivax group where as it was $100 \%, 88.15 \%$ and $46.05 \%$ in falciparum respectively at 24,48 and 72 hours. In a study by Borrmann et al, they found that The proportion of patients with residual parasitemia on day 1 rose from $55 \%$ in $2005-2006$ to $87 \%$ in $2007-2008$ (odds ratio, 5.4, 95\% CI, 2.7-11.1; $\mathrm{P}<0.001)$ and from $81 \%$ to $95 \%$ (OR, 4.1, 95\% CI, 1.7-9.9; $\mathrm{P}=0.002$ ) in the DHA-PPQ and AM-LM groups, respectively. Our results of residual parasitemia were similar to this study again raising concerns about ACT resistance in Indian setup [20].

Many recent studies have shown that funding for the malaria program has decreased due to which significant increase in incidence rate was found in year 2016 as compared to 2015. A continuous monitoring should be setup at all the malaria center and it should be implemented as a part of health policy changes to understand in detail the emerging resistance of Artemisin combined therapy. In our study we used peripheral smear based parasite index as monitoring tool which can be used at the remotest location in the primary health centers too making it easier to monitor [2].

\section{Conclusion}

The study shows that PCT and residual parasitemia is very high in falciparum patients as compared to previous reports of different studies and also as compared to vivax group patients. ACT resistance is a grave concern for falciparum and more studies should be done to understand pathophysiology and its prevalence in India.

Although from our study we can not prove increased resistance of ACT in falciparum patients but on comparing PCT and residual parasitemia with other studies it does show that ACT is getting less effective. Therefore we strongly suggest that a continuous monitoring needs to be implemented in health policy to understand the dynamicity of emerging resistance.

\section{Contributions:}

Chinmay Jani: Contributed in Data Anlaysis, Manuscript writing

Neil Palkhiwala: Contributed in conceiving and designing research question, Data Collection

Ami Parikh: Contributed in conceiving and designing research question, Data Collection

Nilay Suthar: Contributed in conceiving and designing research question, Data Collection, Data Analysis

Khushali Patel: Contributed in conceiving and designing research question, Data Collection.

Funding: Nil, Conflict of interest: None

Permission of IRB: Yes

\section{References}

1. Deribew A, Dejene T, Kebede B, et al. Incidence, prevalence and mortality rates of malaria in Ethiopia from 1990 to 2015: analysis of the global burden of diseases 2015. Malar J. 2017 Jul 4;16(1):271. doi: 10. 1186/s12936-017-1919-4.

2. Bagcchi S. Progress on malaria stalls amid decline in funding. BMJ. 2017 Dec 5;359:j5645. doi: 10.1136/ bmj.j5645.

3. Lufele E, Umbers A, Ordi J, Ome-Kaius M, Wangnapi R, Unger H, et al. Risk factors and pregnancy outcomes associated with placental malaria in a prospective cohort of Papua New Guinean women. Malaria journal. 2017;16(1):427.

4. Guidelines for the treatment of malaria, 3rd ed, WHO.

5. Newton P, White N. Malaria: new developments in treatment and prevention. Annu Rev Med. 1999;50:17992. doi:10.1146/annurev.med.50.1.179

6. Douglas NM, Anstey NM, Angus BJ, et al. Artemisinin combination therapy for vivax malaria. Lancet Infect Dis. 2010 Jun;10(6):405-16. doi: 10.1016/ S1473-3099(10)70079-7.

7. Douglas NM, Nosten F, Ashley EA, et al. Plasmodium vivax recurrence following falciparum and mixed species malaria: risk factors and effect of antimalarial kinetics. Clin Infect Dis. 2011 Mar 1;52(5): 612-20. doi: 10.1093/cid/ciq249. 


\section{Original Research Article}

8. Ashley EA, Dhorda M, Fairhurst RM, Spread of artemisinin resistance in Plasmodium falciparum malaria. N Engl J Med. 2014 Jul 31;371(5):411-23. doi: 10. 1056/NEJMoa1314981.

9. Dondorp AM, Nosten F, Yi P, Artemisinin resistance in Plasmodium falciparum malaria. N Engl J Med. 2009 Jul 30; 361 (5): 455-67. doi: 10.1056/ NEJM oa0808 859.

10. Price RN, Douglas NM, Anstey NM, von Seidlein L. Plasmodium vivax treatments: what are we looking for? Curr Opin Infect Dis. 2011 Dec;24(6):578-85. doi: 10.1097/QCO.0b013e32834c61e3.

11. Chan WW, Virmani D, Pillai DR. Artemisinin combination therapy can result in clinical failure if oral therapy is not directly observed. The Canadian journal of infectious diseases \& medical microbiology = Journal canadien des maladies infectieuses et de la microbiologie medicale. 2013;24(4):215-6.

12. Thita T, Jadsri P, Thamkhantho J, Phenotypic and genotypic characterization of Thai isolates of Plasmodium falciparum after an artemisinin resistance containment project. Malar J. 2018 May 15;17(1):197. doi: 10.1186/s12936-018-2347-9.

13. Singh GP , Goel P, Sharma A. Structural mapping of Kelch13 mutations associated with artemisinin resistance in malaria. J Struct Funct Genomics. 2016 Sep;17(2-3):51-6. doi: 10.1007/s10969-016-9205-1. Epub 2016 Jul 11.
14. Salam N, Mustafa S, Hafiz A, et al. Global prevalence and distribution of coinfection of malaria, dengue and chikungunya: a systematic review. BMC Public Health. 2018 Jun 8;18(1):710. doi: 10.1186/ s12889-018-5626-z.

15. Sehgal PN. Malaria: radical treatment for falciparum malaria in chloroquine resistant strain areas. Health Millions. 1998 Nov-Dec;24(6):18-9.

16. Dash AP, Valecha N, Anvikar AR, Kumar A. Malaria in India: challenges and opportunities. J Biosci. 2008 Nov;33(4):583-92.

17. Sinha S, Medhi B, Sehgal R. Challenges of drugresistant malaria. Parasite. 2014; 21:61. doi: 10.1051/ parasite/2014059. Epub 2014 Nov 18.

18. Parija SC, Praharaj I. Drug resistance in malaria. Indian J Med Microbiol. 2011 Jul-Sep;29(3):243-8. doi: 10.4103/0255-0857.83906.

19. Bharti PK, Shukla MM, Ringwald P, Krishna S, Singh PP, Yadav A, et al. Therapeutic efficacy of artemether-lumefantrine for the treatment of uncomplicated Plasmodium falciparum malaria from three highly malarious states in India. Malaria journal. 2016;15(1):498.

20. Borrmann S, Sasi P, Mwai L, Bashraheil M, Abdallah A, Muriithi S, et al. Declining responsiveness of Plasmodium falciparum infections to artemisininbased combination treatments on the Kenyan coast. PloS one. 2011;6(11):e26005.

How to cite this article?

Jani C, Palkhiwala N, Parikh A, Suthar N, Patel K. Artemisin Combined Therapy in malaria patients: Do we need to search for more?. Int J Med Res Rev 2018; 6(07):348-354. doi:10.17511/ijmrr.2018.i07.02. 